\title{
Niveis Baixos de 25-Hidroxivitamina D (25OHD) em Pacientes com Doença Inflamatória Intestinal e sua Correlação com a Densidade Mineral Óssea
}

\section{artigo original}

\author{
HeVelyn Noemberg de Souza \\ FABIANA LÍGIA LORA \\ Carolina A. Moreira Kulak \\ NÁdILA CECYN PIETSZKOWSKI MAÑAs \\ HeDA M. B. AMARANTE \\ VICTÓRIA Z. COCHENSKI BORBA
}

Serviço de Endocrinologia e Metabologia do Hospital de Clínicas da Universidade Federal do Paraná (SEMPR), Curitiba, PR, Brasil (HNS, CAMK, NCPM, VZCB); Serviço de Gastroenterologia do Hospital de Clínicas do Paraná Curitiba, PR, Brasil (FLL, HMBA).

\section{RESUMO}

Introdução: Pacientes com doenças inflamatórias intestinais (DII) estão propensos a apresentar níveis baixos de vitamina $\mathrm{D}$ (25OHD) e densidade mineral óssea (DMO) diminuída. Objetivo: Verificar o nível de 250HD em jovens com DIl submetidos à avaliação clínica, dosagens bioquímicas rotineiras e medida da DMO de coluna lombar e fêmur, comparando-os com controles saudáveis. Resultados: 39 pacientes com doença de Crohn (DC) $(p=0,003)$ e 37 com retocolite ulcerativa inespecífica (RCUI) $(p<0,001)$ apresentaram níveis mais baixos de $250 H D$ comparados aos controles, $48,5 \%$ dos pacientes com RCUI tinham deficiência de 250HD. A DMO em coluna lombar foi mais baixa nos doentes (DC $p=0,001$; RCUI $p=0,008$ ). A 25OHD correlacionou-se significativamente com a DMO do fêmur total $(r=0,391 ; p=0,027)$ e colo do fêmur $(r=0,384 ; p=0,03)$ na DC. Conclusão: Foram encontrados níveis baixos de $250 H D$ e DMO em jovens com DIl, sugerindo papel importante desta deficiência na patogênese da doença óssea desses pacientes. (Arq Bras Endocrinol Metab. 2008;52/4:684-691)

Descritores: Vitamina D; Osteoporose; Doença inflamatória intestinal; Densidade mineral óssea; Doença de Crohn; Retocolite ulcerativa inespecífica.

\section{ABSTRACT}

Low Levels of 25-Hydroxyvitamin D (25OHD) in Patients with Inflammatory Bowel Disease and its Correlation With Bone Mineral Density.

Patients with inflammatory bowel disease (IBD) are at risk of having vitamin $D$ deficiency (25-OHD) and low bone mineral density (BMD). Objectives: To measure 25OHD in a young group of IBD patients submitted to a clinical evaluation, routine biochemistry and BMD measurement (lumbar spine and proximal femur). Results: 39 Crohn disease (CD) and 37 ulcerative colitis (UC) patients had lower serum levels of 25OHD compared to the control group (CD $p=0,003$; UC $p<0,001$ ), and $48.5 \%$ of the UC patients were $250 H D$ deficient. Lumbar spine BMD was lower in patients than controls (CD $p=0,001 ; \cup C p=$ 0,008 ). In CD patients, serum levels of $250 H D$ were significantly correlated with total femur $(r=0,391 ; p=0,027)$ and femoral neck $(r=0,384 ; p=0,03$ ) BMD. Conclusion: It was found lower levels of $250 H D$ and BMD in young IBD patients compared to normal controls, suggesting an important role of 25OHD deficiency in the pathogenesis of the IBD bone disease. (Arq Bras Endocrinol Metab. 2008;52/4:684-691)

Keywords: Vitamin D; Osteoporosis; Bone mineral density; Inflammatory bowel disease; Crohn Disease; Ulcerative colitis. 


\section{INTRODUÇÃO}

A Vitamina D E SEUs metabólitos hidroxilados formam um sistema responsável por diversas funções, sendo essencial para manutenção do metabolismo do cálcio, permitindo mineralização óssea normal $(1,2)$. O nível sérico de 25-hidroxivitamina D (25OHD) é um indicador funcional confiável do estoque desta vitamina no organismo $(3,4)$.

Baixos níveis de $25 \mathrm{OHD}$ são encontrados em várias populações, como aquelas com baixa exposição à luz solar, idosos e também em portadores de diversas doenças crônicas, como insuficiência renal, doenças pulmonares, epilepsia e lúpus eritematoso sistêmico (5-10). Pacientes com doença inflamatória intestinal (DII), como a retocolite ulcerativa e a doença de Crohn (DC), também estão sujeitos à alteração nos níveis desta vitamina (11).

Na DII existe prevalência aumentada de baixa densidade mineral óssea (DMO) quando comparada a controles saudáveis, com taxas bastante variáveis entre os diversos estudos existentes, em virtude das diferentes definições e populações estudadas. A doença óssea na DII tem causa multifatorial, incluindo inflamação sistêmica, uso crônico de corticosteróides, má nutrição, ressecções intestinais, baixa atividade física e hipogonadismo $(6,8,9$, 12-16). A insuficiência da vitamina D nestes pacientes tem sido descrita por diversos autores e pode contribuir para a diminuição da DMO.

As associações entre baixos níveis de $250 H D$ e diminuição da mineralização óssea são bem reconhecidas em pacientes idosos e em mulheres na pós-menopausa (1,2,9,16-20). Porém, o papel dessa deficiência na patogênese da osteopenia e da osteoporose, freqüentemente encontradas em pacientes com DII, ainda não foi bem definido (14).

O objetivo deste estudo foi avaliar a prevalência de deficiência da vitamina $\mathrm{D}$ na população de pacientes com DII atendida no ambulatório de gastroenterologia do Hospital de Clínicas da Universidade Federal do Paraná (UFPR), correlacionando os níveis de $25 \mathrm{OHD}$ com a DMO e comparando-os a controles saudáveis.

\section{PACIENTES E MÉTODOS}

\section{Pacientes}

Dos 190 pacientes acompanhados no ambulatório de DII do Hospital de Clínicas da UFPR, foram seleciona- dos aqueles com idade entre 20 e 50 anos com diagnóstico de retocolite ulcerativa inespecífica (RCUI) ou DC confirmado por endoscopia, radiologia e histologia antes do início do estudo. Esses pacientes eram convidados a participar do estudo ao realizar sua consulta periódica no ambulatório, quando eram, então, programados os exames.

Foram excluídas todas as mulheres na pós-menopausa, aquelas com ciclos menstruais irregulares ou em terapia estrogênica, os portadores de doenças que causem alterações do metabolismo ósseo, como hepatopatia crônica, hipogonadismo, hipoparatireoidismo, hipertireoidismo ou hipotireoidismo, artrite reumatóide, lúpus eritematoso sistêmico, insuficiência renal crônica, doença pulmonar obstrutiva crônica grave e outras doenças que causem diarréia e má-absorção que não a DII.

As informações foram obtidas por meio de questionário aplicado na consulta e pela revisão de prontuários. Os critérios clínicos avaliados foram: grau de atividade da doença pelo índice de Truelove e Witts para RCUI (21) e BEST para DC (22), tempo de duração da doença, seguimento intestinal comprometido e sua extensão, seguimentos ressecados nos pacientes já submetidos à cirurgia, índice de massa corpórea (IMC), prática de atividade física, tabagismo, etilismo e dose de corticóide do último ano, sendo a dose média diária expressa em miligramas de prednisona.

Foram avaliados 40 controles saudáveis, que apresentavam características homogêneas de idade, sexo, IMC, raça, tabagismo e etilismo com os pacientes estudados e não possuíam nenhum dos critérios de exclusão.

O estudo foi aprovado pelo comitê de ética da instituição e foi obtido consentimento informado escrito dos pacientes e dos controles.

\section{Bioquímica}

A coleta laboratorial foi realizada após 12 horas de jejum, sendo dosados cálcio, fósforo e fosfatase alcalina pelos métodos laboratoriais padrão. Os exames dos pacientes e controles (inclusive a 25OHD) foram colhidos ao longo do ano, de acordo com suas consultas de acompanhamento de rotina. A $25 \mathrm{OHD}$ sérica foi medida usando radioimunoensaio (RIA; DiaSorin, Minessota, EUA), realizado no laboratório Central de Radioimunoensaio de São Paulo (Criesp). O limite de detecção do método era de $1,5 \mathrm{ng} / \mathrm{ml}$ e a variação in- 
tra-ensaio menor que $12,5 \%$ para faixas de detecção entre 8,6 e $49 \mathrm{ng} / \mathrm{ml}$. Neste estudo, considerou-se como deficiência de vitamina $\mathrm{D}$ níveis menores que 20 $\mathrm{ng} / \mathrm{ml}$, insuficiência entre 20 e $30 \mathrm{ng} / \mathrm{ml}$, e normal se maior que $30 \mathrm{ng} / \mathrm{ml}(1,2)$.

\section{Densitometria óssea}

A DMO da coluna lombar e fềmur direito foi medida por um único operador no mesmo dia das coletas laboratoriais. A medida foi realizada por densitômetro de dupla emissão de energia (DXA) em um aparelho Hologic QDR1000/W. Os resultados foram expressos em gramas $/ \mathrm{cm}^{2}$ e o coeficiente de variabilidade foi $0,46 \%$ na coluna lombar e de $0,52 \%$ no fêmur proximal e os desvios-padrão dos valores normais fornecidos pelo valor de referência do aparelho.

A DMO baixa foi definida como a diminuição dela maior que $-2,0$ desvios-padrão da média da população jovem normal ( $\mathrm{Z}$ score), de acordo com as posições oficiais da Sociedade Internacional de Densitometria Clínica (ISCD) (23).

\section{Análise estatística}

Realizou-se a análise descritiva de dados por meio de tabelas com os resultados expressos em média \pm desvios-padrão. Foi realizado um gráfico tipo dispersão para mostrar a correlação significativa entre os valores da DMO no colo de fềmur e da vitamina D nos pacientes com DC (Figura 1). O teste de Kolmogorov-Smirnov foi utilizado para certificar o pressuposto de normalidade dos dados, porquanto somente a dosagem de albumina sérica do grupo DC apresentou distribuição anormal. Para comparação entre os grupos de doentes, foi utilizado o teste $t$ de Student para amostras não-pareadas e com distribuição normal e o teste de Mann-Whitney para dados que não apresentavam essa distribuição. Para comparação entre os dados dos três grupos, DC, retocolite ulcerativa inespecífica e controles, foi utilizada a análise de variância (ANOVA). O coeficiente de correlação de Pearson foi utilizado para correlacionar variáveis numéricas ou nominais para os dados de distribuição normal, e de Spearman para dados que não apresentaram essa distribuição (por meio do software SSPS versão 13.0). O nível de significância adotado foi menor que $5 \%(\mathrm{p}<0,05)$.

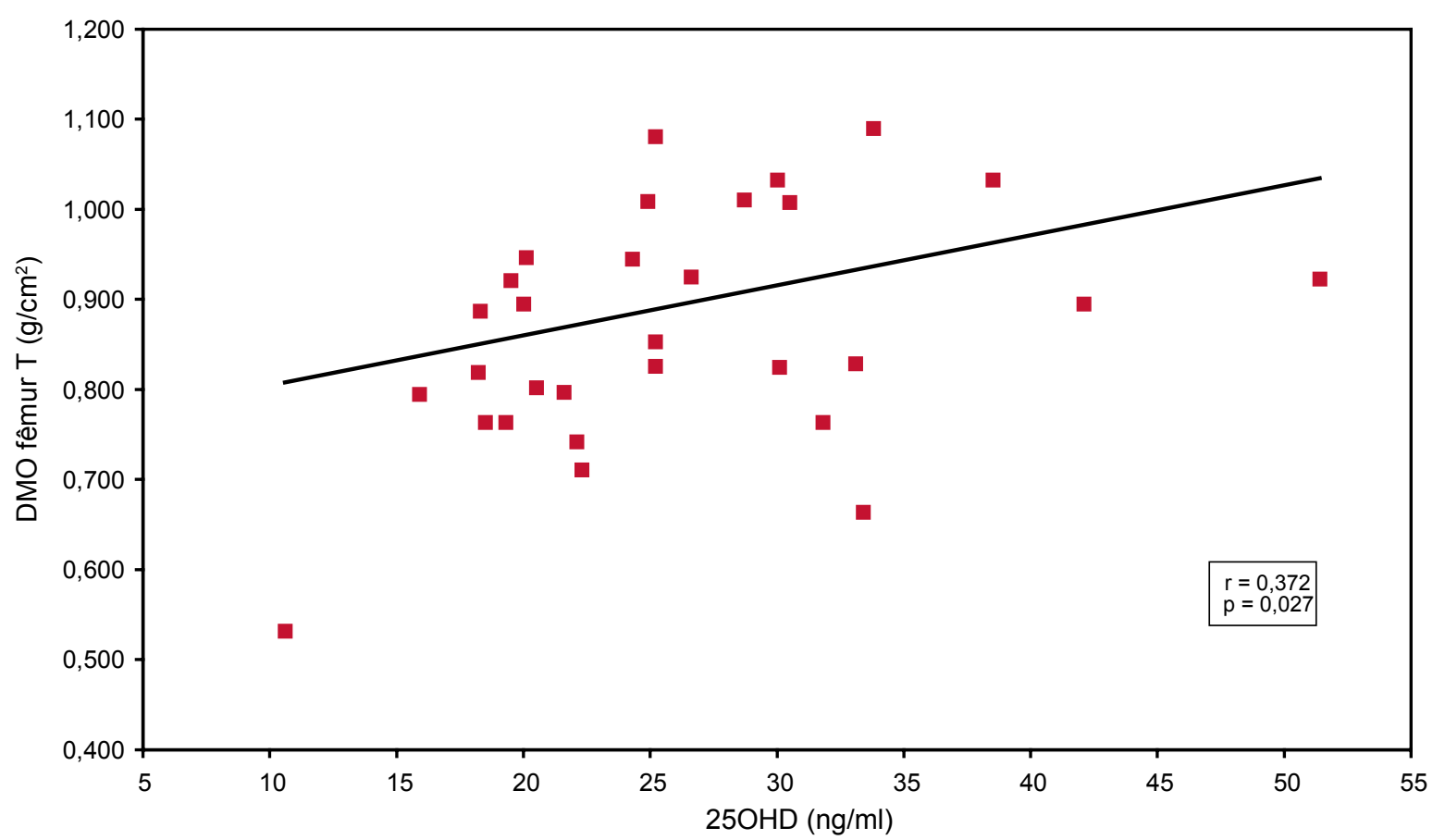

Figura 1. Correlação significativa entre os níveis $250 \mathrm{HD}$ e a DMO do fêmur total $(n=32)$ em pacientes com DC. 


\section{RESULTADOS}

Preencheram os critérios propostos 76 pacientes, 37 com RCUI e 39 com DC. Características descritivas dos pacientes e controles estão na Tabela 1. Os dois grupos de pacientes diferiam na proporção entre homens e mulheres acometidos e cirurgias a que haviam sido submetidos, porquanto 14 pacientes com DC $(35,9 \%)$ e nenhum com RCUI tinham enterectomia prévia. Não houve diferença entre raça, tabagismo, etilismo e atividade física entre os pacientes.

\section{Densidade mineral óssea}

Apresentaram baixa DMO para a idade na coluna lombar oito pacientes com RCUI (22\%) e sete com DC (18\%), sendo significativamente menor nos pacientes com DII do que nos controles (DC p $<0,001$; RCUI $\mathrm{p}=0,008$ na coluna), sem diferença estatística entre as duas doenças. Pacientes com DC apresentavam DMO significativamente menor no colo do fêmur e no fêmur total em relação aos controles (Tabela 2).

Tabela 1. Características clínicas e laboratoriais de pacientes e controles.

\begin{tabular}{|c|c|c|c|}
\hline & DC & RCUI & Controles \\
\hline Número de pacientes & 39 & 37 & 40 \\
\hline Idade (anos) & $32,1 \pm 8,7$ & $35,0 \pm 8,5$ & $34,0 \pm 7,0$ \\
\hline Sexo $(F / M)$ & $18 / 21^{\star}$ & $25 / 12$ & $24 / 16$ \\
\hline $\mathrm{IMC}\left(\mathrm{kg} / \mathrm{cm}^{2}\right)$ & $23,0 \pm 3,6$ & $24,3 \pm 4,0$ & $24,3 \pm 3,9$ \\
\hline Tempo de doença (anos) & $6,3 \pm 4,6$ & $5,6 \pm 3,8$ & - \\
\hline $\begin{array}{l}\text { Grau de atividade da doença } \\
\text { (leve/moderado) }\end{array}$ & $27 / 12$ & $30 / 7$ & - \\
\hline $\begin{array}{l}\text { Dose de corticóide } \\
\text { (mg prednisona/dia) }\end{array}$ & $13,5 \pm 12,1$ & $9,7 \pm 7,1$ & - \\
\hline Cálcio (mg/dl) & $9,1 \pm 0,4$ & $9,0 \pm 0,5$ & $9,1 \pm 0,3$ \\
\hline Fósforo (mg/dl) & $3,7 \pm 0,6$ & $3,5 \pm 0,7$ & $3,8 \pm 0,6$ \\
\hline Albumina (g/dl) & $4,4 \pm 0,3$ & $4,4 \pm 0,3$ & $4,3 \pm 0,4$ \\
\hline Fosfatase alcalina (U/I) & $191 \pm 63$ & $209 \pm 71$ & $100 \pm 36^{\star *}$ \\
\hline $25 \mathrm{OHD}(\mathrm{ng} / \mathrm{ml})$ & $25,9 \pm 8,2$ & $21,8 \pm 8,0$ & $34,4 \pm 12,8^{* * *}$ \\
\hline
\end{tabular}

${ }^{*} \mathrm{p}<0,05$ versus $\mathrm{RCUl} ;{ }^{* *} \mathrm{p}<0,001 \mathrm{DC}$ versus $\mathrm{RCUI} ;{ }^{* \star} \mathrm{p}=0,003$ versus $\mathrm{DC} ; \mathrm{p}<0,001$ versus $\mathrm{RCUl}$.

Tabela 2. Comparação entre a DMO dos pacientes e dos controles em $\mathrm{g} / \mathrm{cm}^{2}$.

\begin{tabular}{|c|c|c|c|c|}
\hline & DC & RCUI & Controles & Valor $p$ \\
\hline \multirow[t]{3}{*}{ DMO coluna lombar } & $0,937 \pm 0,117$ & $0,960 \pm 0,152$ & $1,048 \pm 0,107$ & ${ }^{*}<0,001$ \\
\hline & & & & ${ }^{* *}=0,008$ \\
\hline & & & & ${ }^{* * *}=0,717$ \\
\hline \multirow[t]{3}{*}{ DMO fêmur total } & $0,893 \pm 0,142$ & $0,936 \pm 0,135$ & $0,998 \pm 0,140$ & ${ }^{*}=0,003$ \\
\hline & & & & ${ }^{* *}=0,139$ \\
\hline & & & & ${ }^{* * *}=0,365$ \\
\hline \multirow[t]{3}{*}{ DMO colo do fêmur } & $0,820 \pm 0,131$ & $0,857 \pm 0,134$ & $0,895 \pm 0,119$ & ${ }^{*}=0,028$ \\
\hline & & & & ${ }^{* *}=0,413$ \\
\hline & & & & ${ }^{* * *}=0,417$ \\
\hline
\end{tabular}

* DC versus controle; ** RCUl versus controle; *** DC versus RCUI. 
Não foi encontrada correlação entre a baixa DMO com uso de corticosteróides, sexo, idade, tempo de doença, grau de atividade da doença, atividade física, enterectomia, tabagismo ou etilismo, porém houve correlação significativa entre a DMO em todos os sítios aferidos e o IMC dos pacientes com DII, sendo na coluna lombar $\mathrm{p}=0,005$ e $\mathrm{r}=0,318$, no fêmur total $\mathrm{p}<0,001 \mathrm{e}$ $\mathrm{r}=0,468$ e no colo do fêmur $\mathrm{p}<0,001$ e $\mathrm{r}=0,434$.

\section{Vitamina D}

Os valores de 25OHD foram de 25,9 $\pm 8,2 \mathrm{ng} / \mathrm{ml} \mathrm{nos}$ pacientes com DC, $21,8 \pm 8 \mathrm{ng} / \mathrm{ml}$ nos com RCUI e $34,4 \pm 12,8 \mathrm{ng} / \mathrm{ml}$ nos controles, sendo significativamente menores nos pacientes com DII quando comparados aos controles (DC p $=0,003$ e RCUI p $<0,001$ ), sem diferença entre os pacientes $(\mathrm{p}=0,2)$.

Apenas $28 \%$ dos pacientes com DC e $24 \%$ dos pacientes com RCUI tinham níveis de $25 \mathrm{OHD}$ considerados normais, deficiência de $25 \mathrm{OHD}$ esteve presente em $25 \%$ dos pacientes com DC e $48,5 \%$ dos pacientes com RCUI. Entre os controles, 58,6\% apresentavam níveis de $25 \mathrm{OHD}$ maiores que $30 \mathrm{ng} / \mathrm{ml}$ e apenas $6,9 \%$ eram deficientes.

Houve correlação estatisticamente significativa entre os níveis de 25OHD e a DMO no fêmur total e no colo do fềmur nos pacientes com DC ( $\mathrm{p}=0,027 ; \mathrm{r}=0,391 \mathrm{e}$ $\mathrm{p}=0,029 ; \mathrm{r}=0,386)$, que pode ser vista na Figura 1 .

Não foi verificada correlação dos níveis de 25OHD com o IMC, o grau de atividade da doença, a enterectomia, a dose de corticóide ou a atividade física.

\section{Bioquímica}

Não houve diferença entre os níveis de cálcio, fósforo e albumina entre os controles e o grupo com DII, nem entre os doentes (Tabela 1). O nível de fosfatase alcalina foi significativamente maior nos doentes em relação aos controles $(\mathrm{p}<0,001)$.

Nos pacientes com DC, os níveis de cálcio foram significativamente correlacionados com os níveis séricos de 25OHD ( $\mathrm{p}=0,036 ; \mathrm{r}=0,372)$.

\section{DISCUSSÃo}

Foi encontrado neste grupo de pacientes jovens portadores de DII alta prevalência de insuficiência de vitamina D e de DMO baixa para a idade, quando comparados a controles saudáveis.
Atualmente existe grande debate na literatura sobre quais valores de $250 H D$ devam ser considerados normais, ainda não havendo consenso sobre o valor de corte para a definição de pacientes deficientes, insuficientes ou suficientes em vitamina D. No presente estudo, considerou-se o nível de $30 \mathrm{ng} / \mathrm{ml}$ como valor limítrofe de normalidade, e $20 \mathrm{ng} / \mathrm{ml}$ para deficiência, com base em recente revisão de Holick $(1,2)$. Um maior nível de 25OHD é também defendido por Hollis que considera indivíduos com níveis menores que $32 \mathrm{ng} / \mathrm{ml}$ como deficientes. Heaney, Dawson-Hughes, Vieth e Meunier concordam que $30 \mathrm{ng} / \mathrm{ml}$ é o nível ideal de 25OHD para a supressão do PTH com maior absorção de cálcio e maior DMO, visando a prevenção de fraturas (4,32-34).

Estudos sobre prevalência de insuficiência de vitamina $\mathrm{D}$ nessa população descrevem índices bastante variáveis, entre $2 \%$ e $45 \%$ (8,24-31). Uma das principais causas é a variabilidade dos valores de corte para a definição de deficiência e suficiência de vitamina $\mathrm{D}$, além da diversidade da população estudada quanto a idade, gravidade da doença, cirurgias de ressecção intestinal e uso de corticosteróides. Abitbol e cols. estudaram 84 pacientes com DII, 34 com DC e 50 com RCUI, e consideraram somente cinco $(5,9 \%)$ como deficientes, usando valor de corte de $5 \mathrm{ng} / \mathrm{ml}$. O valor médio de $25 \mathrm{OHD}$ foi de $10,16 \pm 5,37 \mathrm{ng} / \mathrm{ml}$ nos pacientes com RCUI e $16,14 \pm 9,66 \mathrm{ng} / \mathrm{ml}$ nos com DC, sendo significativamente diferente entre os grupos (29). Se considerássemos o valor de $20 \mathrm{ng} / \mathrm{ml}$, a maioria dos pacientes do referido estudo seriam considerados deficientes. Gilman e cols. encontraram que, aproximadamente, $20 \%$ dos pacientes com DC tinham níveis de $25 \mathrm{OHD}$ menores que $16 \mathrm{ng} / \mathrm{ml}(28)$ e Silvennoinen mostrou que $45 \%$ dos pacientes com DC, mas nenhum de seus controles tinham níveis menores que $10 \mathrm{ng} / \mathrm{ml}$ (24).

A causa desta deficiência na DII seria a má-absorção intestinal encontrada nesses pacientes, principalmente com DC, pelo acometimento ileal, pela diminuição da circulação entero-hepática de vitamina $\mathrm{D}$ e ressecções intestinais, freqüentes nessa doença (30). Porém, no presente estudo, encontrou-se prevalência maior de insuficiência em pacientes com RCUI (71,9\% versus $69,7 \%$ ), que teoricamente não teriam essa má-absorção. Essa relação já havia sido encontrada em dois outros estudos $(28,29)$. Entre os fatores que podem explicar esse achado, estão: a má nutrição e a falta de exposição solar, que não foram avaliados no presente estudo. Os valores médios da $25 \mathrm{OHD}$ não foram diferentes entre as duas doenças, mas foram significativamente menores do que dos 
controles. Infelizmente, não se dispõe da dosagem de paratormônio para correlacionar alteração da massa óssea, níveis de vitamina D e hiperparatireoidismo secundário.

A Associação Americana de Gastroenterologia em recente guideline (14) afirma que a incidência de deficiência de vitamina $\mathrm{D}$ não é comum em pacientes com DII e que também não seria causa de diminuição de massa óssea nesses pacientes. Nossos achados discordam dessa afirmação, além de considerar que o estado da vitamina $\mathrm{D}$ deve ser um fator a ser avaliado preventivamente nos pacientes com DII, essencialmente naqueles com DMO diminuída.

A causa da diminuição da DMO em pacientes com DII é multifatorial. Diversos estudos correlacionam essa diminuição a fatores relacionados à doença em si (inflamação sistêmica e má-absorção de cálcio e vitamina $\mathrm{D}$ ), ao seu tratamento (uso de corticosteróides e ressecções intestinais) e às condições associadas a sua morbidade (baixo peso, baixo grau de atividade física, má nutrição, hipogonadismo) (14,26,40-42), sendo difícil separar o papel isolado desses fatores na patogênese da doença óssea na DII.

A prevalência de DMO baixa para a idade foi equivalente entre os dois grupos de pacientes $(22 \%$ RCUI versus $18 \%$ DC na coluna lombar), porém, maior que dos controles $(2,5 \%)$. Estudos prévios, que utilizaram como referência um $\mathrm{Z}$ score menor que $-2,0$, mostraram prevalência bastante variável, entre $2 \%$ e $32 \%$, mas com grande diferença entre os pacientes estudados, principalmente quanto a idade, grau de atividade $\mathrm{e}$ tempo de doença e uso de corticosteróides (34-39). Pode-se considerar essa prevalência ainda mais preocupante se levar em conta o tempo de doença relativamente curto nos pacientes do presente estudo $(6,30 \pm$ 4,60 na DC e 5,60 $\pm 3,80$ na RCUI) e ainda o fato destes pacientes serem adultos jovens. Um fator agravante é que muitos pacientes já apresentam diminuição da DMO e estão em fase de formação do pico de massa óssea. Levando-se em consideração a persistência da doença, reativação freqüente, somadas ainda a fatores já determinados para a diminuição da DMO ao longo da vida, parece preocupante que pacientes jovens apresentem esta diminuição precoce da DMO, que pode contribuir para o aumento do risco de fraturas desses pacientes na senilidade. Lamb e cols. encontraram diminuição da DMO em pacientes com DC com diagnóstico há menos de seis meses (30).
A DMO foi correlacionada significativamente com o IMC, em todos os sítios analisados, com a 25OHD e com o cálcio no fềmur total e no colo do fềmur. Não se localizou nenhum estudo na literatura que tenha encontrado essa correlação entre a $\mathrm{DMO}$ e os níveis de $25 \mathrm{OHD}$ em DII, embora vários estudos tenham tentado evidenciar essa correlação $(8,24-29,31)$. Esse dado vem ressaltar a importância de baixos níveis de vitamina $\mathrm{D}$ na patogênese da doença óssea desses pacientes. No presente estudo, não foi encontrada correlação entre a DMO e a dose de corticosteróides, nem com o seu tempo de uso, já encontrada em diversos outros estudos $(8,9,26,29,35)$. Talvez isso tenha ocorrido por se ter realizado uma avaliação pontual, pois não se dispunha da dose total cumulativa de corticosteróide utilizada por esses pacientes.

Outro achado do presente estudo foi a diferença significativa nos níveis de fosfatase alcalina entre os pacientes com DII e controles. Apesar de não utilizar a fosfatase alcalina óssea-específica, esta diferença pode dar uma idéia indireta de aumento do metabolismo ósseo nestes pacientes. Deve-se considerar que apesar de o aumento da isoforma intestinal da fostatase alcalina poder estar presente em nível tecidual, níveis séricos elevados contribuindo para o aumento da fosfatase alcalina total não foram observados em pacientes com DII (43) e nenhum dos pacientes do presente estudo apresentavam outra causa para essa alteração, como hepatopatia crônica. $\mathrm{Na}$ presente análise, os níveis de fosfatase não se correlacionaram com a DMO em nenhum dos sítios estudados.

\section{CONCLUSÃO}

No presente estudo encontrou-se diminuição da DMO em pacientes jovens com doença inflamatória intestinal e baixos níveis de vitamina $\mathrm{D}$. Houve correlação entre esses dois fatores nos pacientes com doença de Crohn, sugerindo papel importante da deficiência da vitamina $\mathrm{D}$ na patogênese da doença óssea desses pacientes.

\section{REFERÊNCIAS}

1. Holick MF. Resurrection of vitamin D deficiency and rickets. $J$ Clin Invest. 2006;116:2062-72.

2. Holick MF. Vitamin D deficiency. N Engl J Med. 2007; 357:266-81.

3. Hollis BW. Circulating 25-hidroxyvitamin D levels indicative of vitamin D sufficiency: implications for establishing a new effective dietary intake recommendation for vitamin D. J Nutr. 2005;135:317-22 
4. Dawson-Hughes B, Heaney RP, Holick MF, Lips P, Meunier PJ, Vieth R. Estimates of optimal vitamin D status. Osteoporos Int. 2005; 16:713-6.

5. Premaor MO, Furlanetto TW. Vitamin D deficiency in adults: to better understand a new presentation of an old disease. Arq Bras Endocrinol Metab. 2006;50/1:25-37.

6. Kulak CAM, Borba VZC, Bilezikian JP, Silvado CE, De Paola L, Boguszewski CL. Bone mineral density and serum levels of 25 $\mathrm{OH}$ vitamin D in chronic users of antiepileptic drugs. Arq Neuropsiquiatr. 2004;62(4):940-8.

7. Vieira JG, Katmasu T, Radominski SC, Sato El, Lazaretti-Castro M. Vitamin D status, bone metabolism and cytokines in patients with active systemic lupus erythematosus. San Francisco: The Endocrine Society Press; 2002.

8. Tajika M, Matsuura A, Nakamura T, Suzuki T, Sawaki A, Kato T, et al. Risk factors for vitamin $D$ deficiency in patients with Crohn's disease. J Gastroenterol. 2004;39:527-33.

9. Sentongo TA, Semaeo EJ, Stettler N, Piccoli DA, Stallings VA, Zemel BS. Vitamin D status in children, adolescents, and young adults with Crohn disease. Am J Clin Nutr. 2002;76:1077-81.

10. Compston JE. Vitamin D deficiency: time for action. BMJ. 1998;317:1466-7.

11. Harpavat M, Keljo DJ, Regueiro MD. Metabolic bone disease in inflammatory bowel disease. J Clin Gastroenterol. 2004;38(3):218-24

12. Bernstein CN, Leslie WD. Review article: osteoporosis in inflammatory bowel disease. Aliment Pharmacol Ther. 2004;19:941-52.

13. Bjarnason I, Macpherson A, Mackintosh C, Buxton-Thomas M, Forgacs I, Moniz C. Reduced bone density in patients with inflammatory bowel disease. Gut. 1997;40:228-33.

14. Bernstein CN, Leslie WD, Leboff MS. AGA technical review on osteoporosis in gastrointestinal diseases. Gastroenterology. 2003;124(3):795-841.

15. Lora FL, Amarante HMB, Pisani JC, Borba VZC, Kulak CAM, Carmes ER. Bone mineral density evaluation in inflammatory bowel disease patients. Arq Gastroenterol. 2005;42(4):201-5.

16. Dawson-Hughes B, Harris SS, Krall EA, Dallal GE. Effect of calcium and vitamin $D$ supplementation on bone density in men and women 65 years of age or older. $\mathrm{N}$ Engl $\mathrm{J}$ Med. 1997;337:670-6.

17. Thomas MK, Lloyd-Jones DM, Thadhani RI, Shaw AC, Deraska DJ, Kitch BT, et al. Hypovitaminosis D in medical inpatients. N Engl J Med. 1998;338:777-83.

18. Papadimitropoulos E, Wells G, Shea B, Gillespie W, Weaver B, Zytaruk N, et al. Meta-analysis of therapies for postmenopausal osteoporosis. VIII. Meta-analysis of the efficacy of vitamin D treatment in preventing osteoporosis in postmenopausal women. Endocr Rev. 2002;23:560-9.

19. Chapuy MC, Arlot ME, Duboeuf F, Brun J, Crouzet B, Arnaud S, et al. Vitamin D3 and calcium to prevent hip fractures in elderly women. N Engl J Med. 1992;327:1637-42.

20. Bischoff-Ferrari HA, Willett WC, Wong JB, Giovannucci E, Dietrich $T$, Dawson-Hughes $B$. Fracture prevention with vitamin $D$ supplementation: a meta-analysis of randomized controlled trials. JAMA. 2005;293:2257-64.

21. Truelove SC, Witts LJ. Cortisone in ulcerative colitis. Final report on a therapeutic trial. Br Med J. 1955;2:1041-8.

22. Best WR, Becktel JM, Singleton JW. Rederived values of eight coefficients of Crohn disease activity index. Gastroenterology. $1979 ; 77: 843-6$
23. Leslie WD, Adler RA, El-Hajj Fuleihan G, Hodsman AB, Kendler $\mathrm{DL}$, McClung $\mathrm{M}$, et al.; International Society for Clinical Densitometry. Application of the 1994 WHO classification to populations other than postmenopausal Caucasian women: the 2005 ISCD Official Positions. J Clin Densitom. 2006;9(1):22-30.

24. Silvennoinen J. Relationships between vitamin D, parathyroid hormone and bone mineral density in inflammatory bowel disease. J Intern Med. 1996;239(2):131-7.

25. Abreu MT, Kantorovivich V, Vasiliauskas EA, Gruntmanis U, Matuk R, Daigle K, et al. Measurement of vitamin D levels in inflammatory bowel disease patients reveals a subset of Crohn disease patients elevated 1,25-dihydroxyvitamin $D$ and low bone mineral density. Gut. 2004;53:1129-36.

26. Ardizzone S, Bollani S, Bettica P, Bevilacqua M, Molteni P, Poro $\mathrm{G}$, et al. Altered bone metabolism in inflammatory bowel disease: there is a difference between Crohn disease and ulcerative colitis. J Intern Med. 2000;247(1):63-70.

27. Jahnsen J, Falch JA, Mowinckel P, Aadland E. Vitamin D status, parathyroid hormone and bone mineral density in patients with inflammatory bowel disease. Scand J Gastroenterol. 2002;37(2):192-9.

28. Gilman F, Shanahan F, Cashman KD. Altered levels of biochemical indices of boné turnover and bone-related vitamins in patients with Crohn disease and ulcerative colitis. Aliment Pharmacol Ther. 2006;23:1007-16.

29. Abitbol V, Roux C, Chaussade S, Guilemant S, Kolta S, Douga$\operatorname{dos} M$, et al. Metabolic bone assessment in patients with inflammatory bowel disease. Gastroenterology. 1995;108:417-22.

30. Lamb EJ, Wong T, Smith DJ, Simpson DE, Coakley AJ, Moniz $C$, et al. Metabolic bone disease is present at diagnostic in patients with inflammatory bowel disease. Aliment Pharmacol Ther. 2002;16:1895-902.

31. McCarthy D, Duggan P, O'Brien M, Kiely J, McCarthy J, Shanahan $F$, et al. Seasonality of vitamin D status and bone turnover in patients with Crohn disease. Aliment Pharmacol Ther. 2005;21:1073-83.

32. Bischoff HA, Stähelin HB, Dick W, Akos R, Knecht M, Salis C, et al. Effects of vitamin $D$ and calcium supplementation on falls: $a$ randomized controlled trial. J Bone Miner Res. 2003;18:343-51.

33. Holick MF. The vitamin D epidemic and its health consequences. J Nutr. 2005;135:S2739-48.

34. Heaney RP. Vitamin D depletion and effective calcium absorption. J Bone Miner Res. 2003;18:1342(letter).

35. Bernstein CN, Seeger LL, Sayre JW, Anton PA, Artinian L, Shanahan $F$. Decreased bone density in inflammatory bowel disease is related to corticosteroid use and not disease diagnosis. J Bone Miner Res. 1995;10:250-6.

36. Pigot F, Roux C, Chaussade S, Hardelin D, Pelleter O, Du Puy $\mathrm{MT}$, et al. Low bone mineral density in patients with inflammatory bowel disease. Dig Dis Sci. 1992;37:1396-403.

37. Roux C, Abitbol V, Chaussade S, Kolta S, Guillemant S, Douga$\operatorname{dos} \mathrm{M}$, et al. Bone loss in patients with inflammatory bowel disease: a prospective study. Osteoporos Int. 1995;5:156-60.

38. Clements D, Motley RJ, Evans WD, Harries AD, Rhodes J, Coles RJ, et al. Longitudinal study of cortical bone loss in patients with inflammatory bowel disease. Scand J Gastroenterol. 1992;27:1055-60.

39. Compston JE, Judd D, Crawley EO, Evans WD, Evans C, Church $\mathrm{HA}$, et al. Osteoporosis in patients with inflammatory bowel disease. Gut. 1987;28:410-5.

40. Lin CL, Moniz C, Chambers TJ, Chow JW. Colitis cause bone loss in rats through suppression of bone formation. Gastroenterology. 1996;111:1263-71. 
41. Hyams JS, Wysga N, Kreutzer DL, Justinich CJ, Gronowicz GA Alterations in bone metabolism in children with bowel inflammatory disease. J Pediatr Gastroenterol Nutr. 1997;24:289-95.

42. Pollak RD, Karmeli F, Eliakim R, Ackerman Z, Tabb K, Rachmilewitz D. Femoral neck osteopenia in patients with inflammatory bowel disease. Am J Gastroenterol. 1998;93:1483-90.

43. Domar U, Danielsson A, Hirano K, Stigbrand T. Alkaline Phosphatase isoenzymes in non-malignant intestinal and hepatitic diseases. Scand J Gastroenterol. 1988;23:793-800.
Endereço para correspondência:

Victória Z. Cochenski Borba

Av. Agostinho Leão Júnior, 285, Alto da Glória 80030-110 Curitiba, PR

E-mail: familiabborba@terra.com.br 